\title{
The effects of COVID-19 on mental health of the society: A dynamic approach in Iran
}

\author{
Alireza Raesi ${ }^{1}$, Ahmad Hajebi*2 (D) , Maryam Rasoulian ${ }^{3}$, Maryam Abbasinejad $^{4}$ \\ Received: 29 May 2020 \\ Published: 20 Aug 2020 \\ Conflicts of Interest: None declared \\ Funding: None \\ *This work has been published under CC BY-NC-SA 1.0 license. \\ Copyright $\subseteq$ Iran University of Medical Sciences
}

Cite this article as: Raesi A, Hajebi A, Rasoulian M, Abbasinejad M. The effects of COVID-19 on mental health of the society: A dynamic approach in Iran. Med J Islam Repub Iran. 2020 (20 Aug);34:102. https://doi.org/10.47176/mjiri.34.102

On March 11, 2020, the World Health Organization (WHO) declared the outbreak of a new coronavirus disease, COVID-19, to be a pandemic (1) and called for countries to take urgent and aggressive action (2). The world was faced with a new crisis that not only involved the health sector but also several other sectors. ${ }^{2}$ Confirmed COVID19 cases have exceeded 4.5 million globally, with confirmed deaths going over 283000 , according to the latest official figures derived from official WHO data on May 13, 2020 (3). The Islamic Republic of Iran announced its first COVID-19 positive case on February 20, 2020 (4). The stressful atmosphere observed in societies previously infected by the virus also gradually reached Iran, and in most of the cities, it was even more intense than the real stress related to the virulence of the disease (5). Paying attention to the mental health of societies struck by a crisis has been shown to be one of the fundamental aspects of crisis management programs worldwide (6-8). The Islamic Republic of Iran has more than 2 decades of experience in providing psychosocial support in disasters. Lessons learned from the

Corresponding author:Dr Ahmad Hajebi, hajebi.a@iums.ac.ir

1. Deputy of Public Health, Ministry of Health and Medical Education, Tehran, Iran

2. Research Center for Addiction and Risky Behaviors, Psychiatric Department, Iran University of Medical Sciences, Tehran, Iran

3. Mental Health Research Center, Tehran Institute of Psychiatry, Faculty of Behavioral Sciences and Mental Health, Iran University of Medical Sciences, Tehran, Iran

4. Department for Mental Health and Substance Abuse, Ministry of Health and Medical Education, Tehran, Iran management of mental health issues in emergencies, especially during the COVID-19 pandemic, revealed to us that in order to efficiently respond to the mental health needs of a society at different stages of a crisis, a dynamic approach has to be adopted.

The rapid spread of the novel coronavirus has brought about a great deal of stress, anxiety, and fear to all societies. These emotions can be considered normal regarding the fact that people are being faced with an unstable, unpredictable and ambiguous situation. Nevertheless, severe and prolonged anxiety may lead to psychological distress, and this in turn will lead to malfunctioning in daily living. High stress levels have been shown to weaken the human immunity system, and therefore lead to vulnerability towards viral infections (9). Stress may also lead to maladaptive behavior. At this point, the Ministry of Health and Medical Education (MOH) of Iran became preoccupied with developing strategies to reach a comprehensive solution for properly addressing the quickly-spreading stress and tension across the society. The mental health staff of the pri-

$\uparrow$ What is "already known" in this topic:

- Addressing mental health issues of the populations adversely affected by a crisis is a fundamental component in the field of crisis management programs worldwide.

- The rapid spread of the novel coronavirus has brought about a great deal of stress, anxiety, and fear to all societies.

$\rightarrow$ What this article adds:

- Lessons learned from the management of mental health issues of the society during the COVID-19 pandemic, showed that in order to efficiently respond to the mental health needs of a society at different stages of a crisis, a dynamic approach has to be adopted, delivered in a timely manner and minimal delay. 
mary health care (PHC) system were especially trained according to developed training packages to provide necessary care for those dealing with stress, and the MOH helpline was also set up in the very beginning of this phase to enhance access to care.

Field reports show that the main emotions observed among Iranian people in the beginning of the virus outbreak were anxiety, worry, and confusion (10). Qualitative analysis of data extracted from the $\mathrm{MOH}$ helpline revealed that most of these emotions were results of low awareness or misinformation related to the disease and virus behavior, standard methods of protection, and stress around shortage of supplies in the beginning of the outbreak (10). This guided the $\mathrm{MOH}$ towards a multimodal approach with the cooperation of other governmental sectors, including the Islamic Republic of Iran Broadcasting System (IRIB). Virtual social networks were also brought into use regarding this approach. Main goals at this phase were changing attitudes of the general population towards different aspects of the COVID-19 pandemic and promoting awareness leading to healthy behavior.

Two to 3 weeks after the announcement of the first cases in Iran, the number of those diagnosed with the disease gradually started to increase. Unfortunately, social stigma surprisingly started to emerge as one of the problems that those directly affected by the virus had to cope with. Therefore, COVID-19 patients and their families not only had to deal with consequences and problems of the disease itself, but also had to fight with the mental burden of being stigmatized by the society. This brought up the need for designing an intervention to increase mental health literacy and decrease the stigma surrounding the disease. Meanwhile at this stage, inpatient services quickly started to become overloaded to such an extent that the medical staff had to work for continuous hours and days giving service to patients in hospitals. This indeed threatened their mental health in several ways. They were going on heavy and continuous working shifts with minimal rest on one hand, and having less social interaction with friends and family members due to protective measures on the other, while continuously experiencing severe anxiety about getting infected due to exposure to the virus. We felt the need to design interventions to specifically address the mental health of medical staff countrywide and respond to their need for psychosocial support. Governmental and nongovernmental organizations worked alongside faculty members across medical universities, especially mental health professionals, to design psychosocial support interventions for service providers.

With the upcoming of the New Year holidays and the intensification of quarantine measures, major changes started to become evident in daily routines of families. To prevent probable conflicts due to these changes and to help individuals maintain effective communication among their family members, we needed to design interventions to primarily increase the awareness of the general population on the necessary skills in this new situation. Some cases were also in need of counselling services offered by mental health professionals. Promoting self-care in the field of mental health in lockdown situations was the main scope of our interventions throughout this period and providing psychological consultations and support via the $\mathrm{MOH}$ helpline was therefore initiated at this stage.

Death tolls of COVD-19 slowly began to rise in the last week of March 2020 in Iran, and at the same time safety health protocols hindered the possibility of traditional burial ceremonies and social gatherings in memory of those deceased. This gave rise to a new critical issue: the necessity to make efforts to prevent complicated grief among COVID-19 survivors. We had to officially advise divisions in charge of safe burial to be considerate towards emotional, cultural, and religious matters along with keeping up with safety protocols. The more important component of this intervention was to provide face-to-face psychological consultation services through the PHC system for those who had experienced loss of loved ones.

Content analysis of calls to the $\mathrm{MOH}$ helpline demonstrate the reality that the stressful atmosphere that had taken over the society in the beginning of the pandemic has faded out now, but social and financial stressors due to the adverse effects of COVD-19 on national economies has presumably resulted in an increase in feelings of despair and hopelessness across the society and in some cases even suicidal ideations.

Due to rapid virus transmission, the COVID-19 pandemic has placed all countries in a state of tension that will subsequently lead to various problems across several aspects of everyday living of human beings across the globe. These problems will in turn lead to psychological distress. Following our 3-month experience in managing the individual and non-individual effects of COVID-19 on mental health of the society in Iran, we have concluded that: (1) Manifestations of psychological distress differs in quality and severity over time and across different stages of the COVD-19 pandemic. (2) Psychosocial interventions must be offered in a timely manner and with minimal delay, because failure to keep up with the immediate needs of the society may lead to complicated issues and consequences difficult to compensate for later on. (3) Designed psychosocial interventions should be flexible and dynamic in nature to be able to respond to the evolving needs of the society.

\section{Conflict of Interests}

The authors declare that they have no competing interests.

\section{References}

1. World Health Organization (2020), https://www.who.int/dg/speeches/ detail, Access. 05.13.2020.

2. Cucinotta D, Vanelli M .WHO Declares COVID-19 a Pandemic. Acta Biomed. 2020 Mar 19;91(1):157-160.

3. World Health Organization (2020), https://www.who.int/emergencies/ diseases/novel-coronavirus-2019, Access. 05.13.2020.

4. Ministry of Health and Medical Education of the Islamic Republic of Iran (2020), http://behdasht.gov.ir, Access. 05.13.2020.

5. Torales J, O'Higgins M, Castaldelli-Maia JM, Ventriglio A. The outbreak of COVID-19 coronavirus and its impact on global mental health. Int J Soc Psychiatry. 2020 Mar 31:20764020915212.

6. Sundram S, Karim ME, Ladrido-Ignacio L, Maramis A, Mufti KA, Nagaraja D, et al, Psychosocial responses to disaster: An Asian perspective. Asian J Psychiatr. 2008 Sep;1(1):7-14.

7. Rao K. Psychosocial support in disaster-affected communities. Int Rev 
Psychiatry. 2006 Dec;18(6):501-5.

8. Dong L, Bouey J. Public Mental Health Crisis during COVID-19 Pandemic, China. Emerg Infect Dis. 2020 Mar 23;26(7).

9. Dhabhar FS. Effects of stress on immune function: the good, the bad, and the beautiful. Immunol Res. 2014 May;58(2-3):193-210.

10. Ministry of Health and Medical Education of the Islamic Republic of Iran, Content analysis of reported problems via the $\mathrm{MOH}$ helpline during the COVID-19 outbreak in Iran. 2020. 